\title{
IDENTIFICACIÓN DE ÁREAS DE POTENCIAL CONFLICTO ENTRE USOS DEL SUELO EN LA CUENCA DEL RÍO LUJÁN, ARGENTINA
}

\section{IDENTIFICATION OF POTENTIAL AREAS OF LAND- USE CONFLICT IN THE LUJAN RIVER BASIN, ARGENTINA}

\author{
Gustavo D. Buzai ${ }^{1}$ \\ Noelia Principi ${ }^{2}$ \\ Universidad Nacional de Luján, Argentina
}

http://dx.doi.org/10.15359/rgac.3-59.4

\section{RESUMEN}

La Geografía, como ciencia espacial, se apoya en la utilización de métodos cuantitativos aplicados con Sistemas de Información Geográfica (SIG). En este contexto, el modelado cartográfico y las técnicas de evaluación multicriterio permitieron definir una estrategia metodológica para la identificación de áreas de potencial conflicto entre usos del suelo en escala regional (LUCIS, Land-use Conflict Identification Strategy). La investigación se realiza en la cuenca del río Luján, con el objetivo de definir estas áreas como consecuencia de la futura competencia espacial entre el uso

1 Prof. Lic. (Geografía, UBA), Dr. (Geografía, UNCuyo) y Estadías PosDr (UAM España, UIBK Austria). Universidad Nacional de Luján y Consejo Nacional de Investigaciones Científicas y Técnicas (CONICET). Instituto de Investigaciones Geográficas (INIGEO). Director del Programa de Docencia e Investigación en Sistemas de Información Geográfica (PRODISIG). Argentina. buzai@unlu.edu.ar

2 Prof. Lic. (Geografía, UNLu) y Mg. (Sistemas de Información Geográfica y Teledetección, UCR-UNA, Costa Rica). Universidad Nacional de Luján. Instituto de Investigaciones Geográficas (INIGEO). Coordinadora del Laboratorio de Análisis Espacial y Sistemas de Información Geográfica (LabSIG). Argentina. nprincipi@unlu.edu.ar 
Gustavo D. Buzai, Noelia Principi. Identification of potential areas of land-use conflict in the Lujan river basin, Argentina

del suelo urbano, la producción agrícola y las áreas de conservación. Los resultados obtenidos constituyen una excelente herramienta de utilidad en la planificación territorial.

Palabras clave: Geografía Aplicada, Análisis Regional, SIG, Evaluación multicriterio, LUCIS

\begin{abstract}
Geography, as spatial science, relies on the use of quantitative methods applied with Geographical Information Systems (GIS). In this context, cartographic modeling and multi-criteria evaluation techniques allowed the definition of a methodological strategy for identifying areas of potential conflict between land-uses at a regional scale (LUCIS). The research is carried out in the Luján river basin with the purpose of defining these areas as a consequence of future spatial competition between urban land-use, agricultural production and conservation areas. The results obtained are an excellent tool to use in the field of land-use planning.
\end{abstract}

Keywords: Applied Geography, Regional Analysis, GIS, Multi-criteria Evaluation, LUCIS

\title{
Introducción
}

Este trabajo analiza aspectos teórico-metodológicos que permiten la identificación de áreas con potencial conflicto ante la evolución espacial del uso del suelo ${ }^{3}$, y realiza su aplicación a la cuenca del río Luján. Se detallan las técnicas de evaluación multicriterio, aplicadas en Sistemas de Información Geográfica (SIG) y la estrategia de resolución denominada Land Use Conflict Identification Strategy (LUCIS). El camino metodológico se sustenta conceptualmente en el materialismo sistémico (Bunge, 2012, 2014) y en el análisis del nivel espacial como focalización realizada en el marco de la teoría de los sistemas complejos. La construcción de una totalidad organizada resulta posible al modelar la realidad en capas temáticas, en un proceso de diferenciación y combinación a partir del modelado cartográfico en la búsqueda de resultados parciales y una síntesis integradora de la situación espacial.

Los procedimientos metodológicos detallados a lo largo de la presentación fueron aplicados inicialmente en Buzai y Baxendale (2007, 2008), donde se ha demostrado que constituyen una excelente alternativa para el estudio prospectivo del espacio geográfico. La identificación de estructuras espaciales permite definir áreas de potencial conflicto como

3 Se utiliza el término uso del suelo por ser reconocido institucionalmente en la República Argentina. El espacio geográfico comprendido por la cuenca del río Luján se encuentra regulado por el Decreto-Ley 8912/77 LEY DE ORDENAMIENTO TERRITORIAL Y USO DEL SUELO (Texto Ordenado por Decreto $3389 / 87$ con las modificaciones del Decreto-Ley $N^{\circ} 10128$ y las Leyes $N^{\circ} 10653,10764,13127,13342$ y 14449) del Ministerio de Gobierno de la Provincia de Buenos Aires. 
consecuencia de la futura competencia espacial entre la expansión urbana, la producción agrícola y las áreas de conservación.

De esta manera, el resultado obtenido constituye una herramienta en apoyo al proceso de toma de decisiones espaciales en el ámbito de la planificación para la generación de propuestas y en la gestión para la posible implementación, siempre en la búsqueda de caminos que lleven a una evolución espacial armoniosa de los usos del suelo (Buzai y Baxendale, 2010, 2011; UF, 2014). La teoría y metodología aplicada simplifica su descripción y análisis en una focalización espacial central en el contexto de la Geografía Aplicada de perspectiva sistémica.

\section{Área de estudio \\ Cuenca hidrográfica del río Luján}

El área de estudio es la cuenca hidrográfica del río Luján, que es en realidad una subcuenca, perteneciente a la Gran Cuenca del Plata. Tiene una superficie de $3761 \mathrm{~km}^{2}$ y sobre ella se encuentran ubicados varios municipios del sector noroeste del Gran Buenos Aires. Al norte limita con las cuencas del arroyo de la Cruz y del río Areco; al oeste y al sur con la cuenca del río Salado y al sureste con la cuenca del río Reconquista.

La delimitación de la cuenca del río Luján fue realizada por Lanzelotti y Buzai (2015) a partir de ajustar los límites definidos en Buzai (2002) y Paso Viola (2013) mediante la utilización de imágenes satelitales SRTM y ASTERGDEM, corregidas mediante interpretación visual.

Los municipios ${ }^{4}$ que integran la cuenca son: Campana, Escobar, Exaltación de la Cruz, General Rodríguez, José C. Paz, Luján, Malvinas Argentinas, Mercedes, Moreno, Pilar, San Andrés de Giles, Carmen de Areco, Chacabuco, San Fernando, Suipacha y Tigre.

La topografía general de la cuenca es suave, las máximas alturas se localizan en el oeste, llegando aproximadamente a los $60 \mathrm{msnm}$, y descendiendo en sentido sureste hasta alcanzar el nivel del mar.

De acuerdo al Instituto Nacional del Agua (2007) la cuenca puede dividirse en tres tramos (Figura 1): la cuenca superior que se extiende desde las nacientes hasta el municipio de Luján, específicamente en la

4 En la República Argentina las divisiones político-administrativas son de las siguientes magnitudes: 1er. Orden (País), 2do. Orden (Provincia), 3er. Orden (Departamento o Partidos en la Provincia de Buenos Aires, donde coinciden espacialmente con el municipio). A partir de aquí son divisiones censales: 4to. Orden (Fracciones censales) y 5to. Orden (Radios censales). 
localidad de Jáuregui, con una pendiente media del tramo de unos $0,45 \mathrm{~m} /$ $\mathrm{km}$; la cuenca media, se extiende hasta el municipio de Pilar, coincidiendo con el cruce del río con la Ruta Nacional $\mathrm{N}^{0}$ 8, con una pendiente media del tramo de $0,36 \mathrm{~m} / \mathrm{km}$, y finalmente la cuenca inferior, se extiende hasta el municipio de Tigre, donde el río Luján desemboca en el Delta del Paraná, poco después del cruce de la Ruta Nacional $N^{\circ} 9$, cuya pendiente media es de $0,04 \mathrm{~m} / \mathrm{km}$.

El curso fluvial principal de la cuenca es el río Luján, que realiza su recorrido por la planicie ondulada de la Pampa, nace en de la confluencia del arroyo de Los Leones y del Durazno y la Cañada de Castilla, en el municipio de Carmen de Areco, y una pequeña superficie del municipio de Chacabuco, con un recorrido en dirección sudoeste-noreste de 115 kilómetros hasta su desembocadura en el delta del Paraná, y luego gira en dirección sudeste y recorre $42 \mathrm{~km}$ hasta llegar al Río de la Plata.

El río Luján se presenta como hidrografía de llanura con escasa pendiente, siendo que, de acuerdo a Paso Viola (2016), sus cotas fluctúan entre los 46 y $58 \mathrm{~m}$ con una media del tramo superior que ronda los $47 \mathrm{~m}$. A través de su recorrido se pueden apreciar paisajes predominantemente urbanos (Figuras 2 y 3), rurales (Figura 4) y seminaturales (Figura 5), como usos del suelo que presentan la base para la identificación de potenciales conflictos espaciales. 


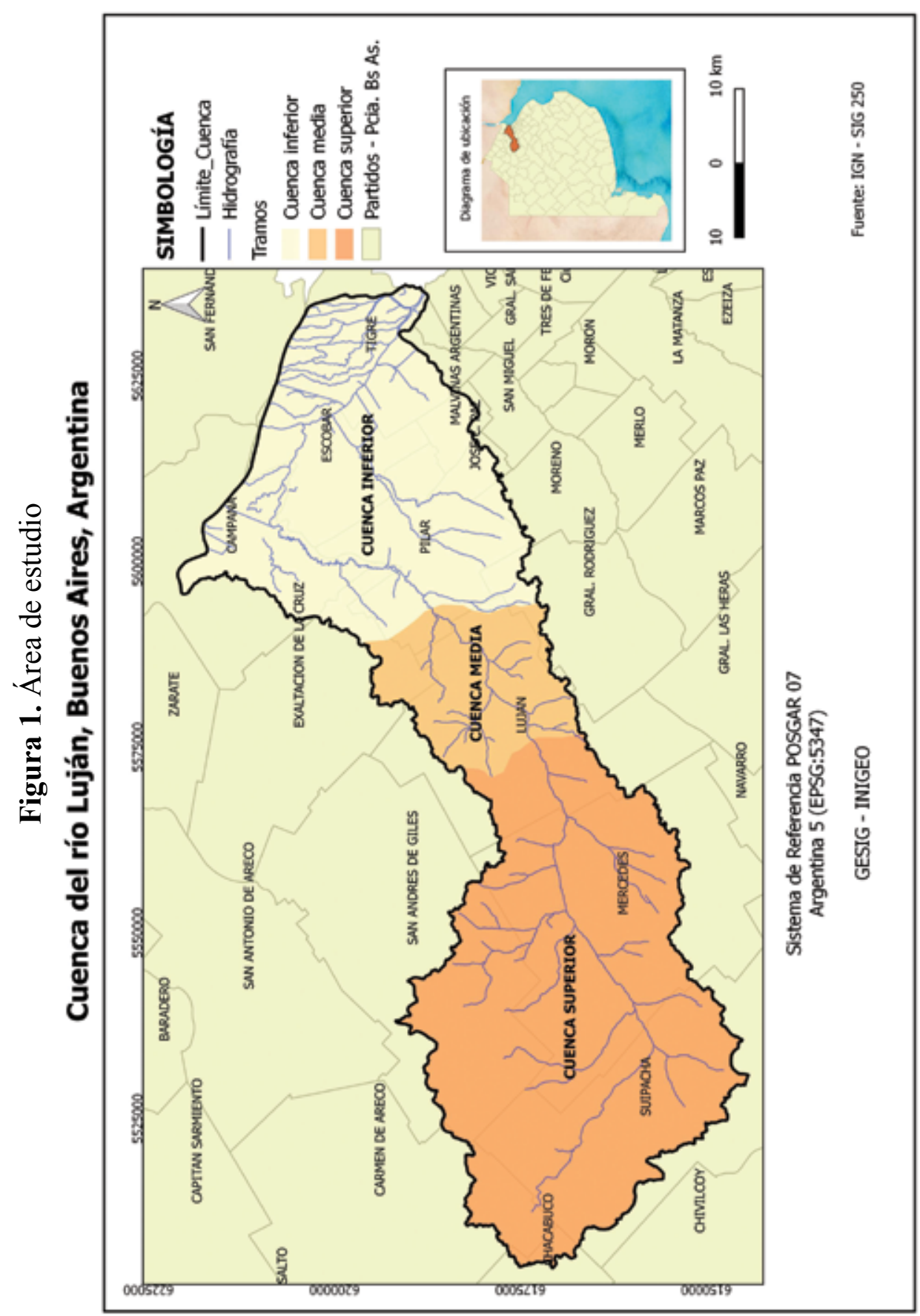


Gustavo D. Buzai, Noelia Principi. Identification of potential areas of land-use conflict in the Lujan river basin, Argentina

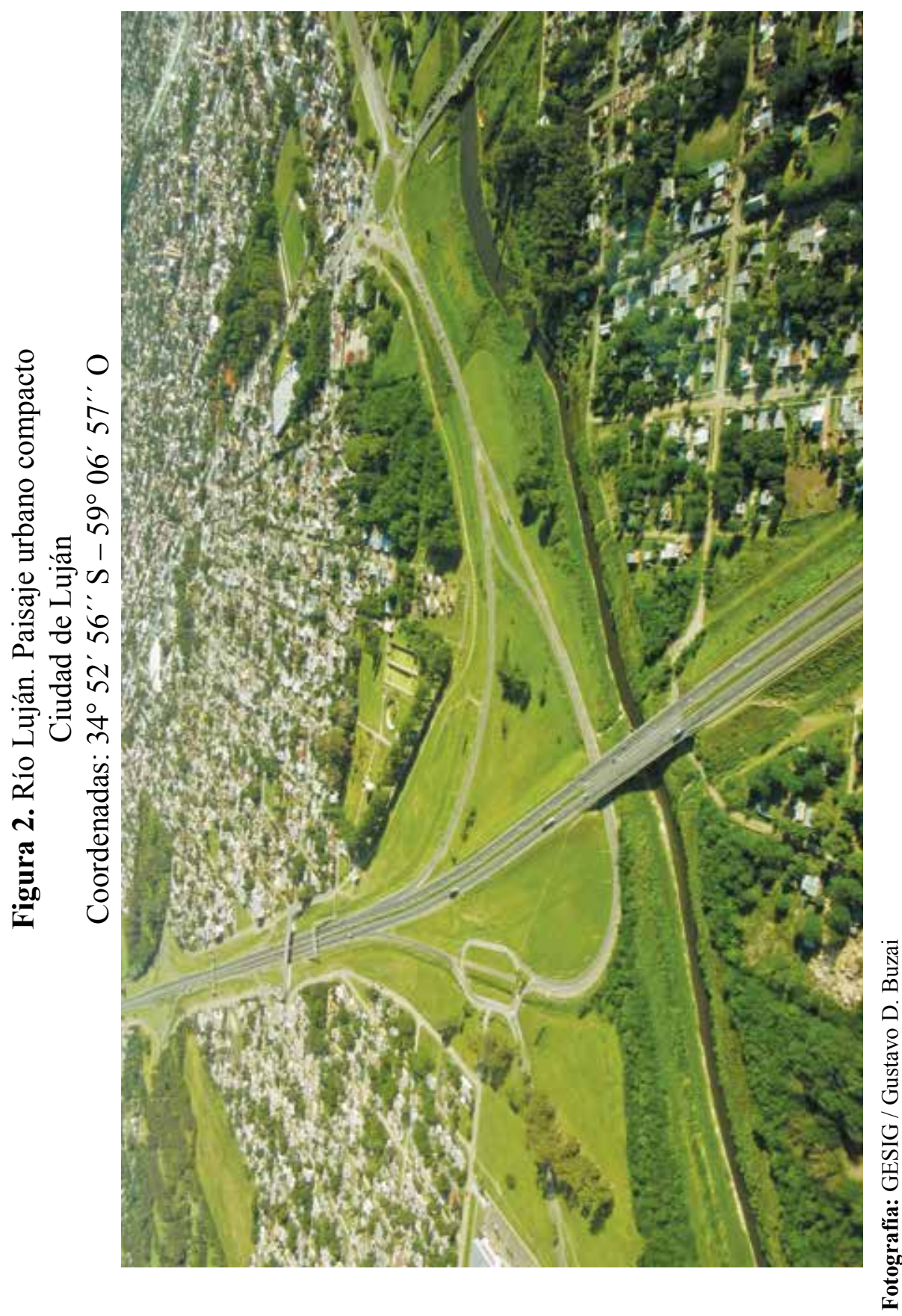




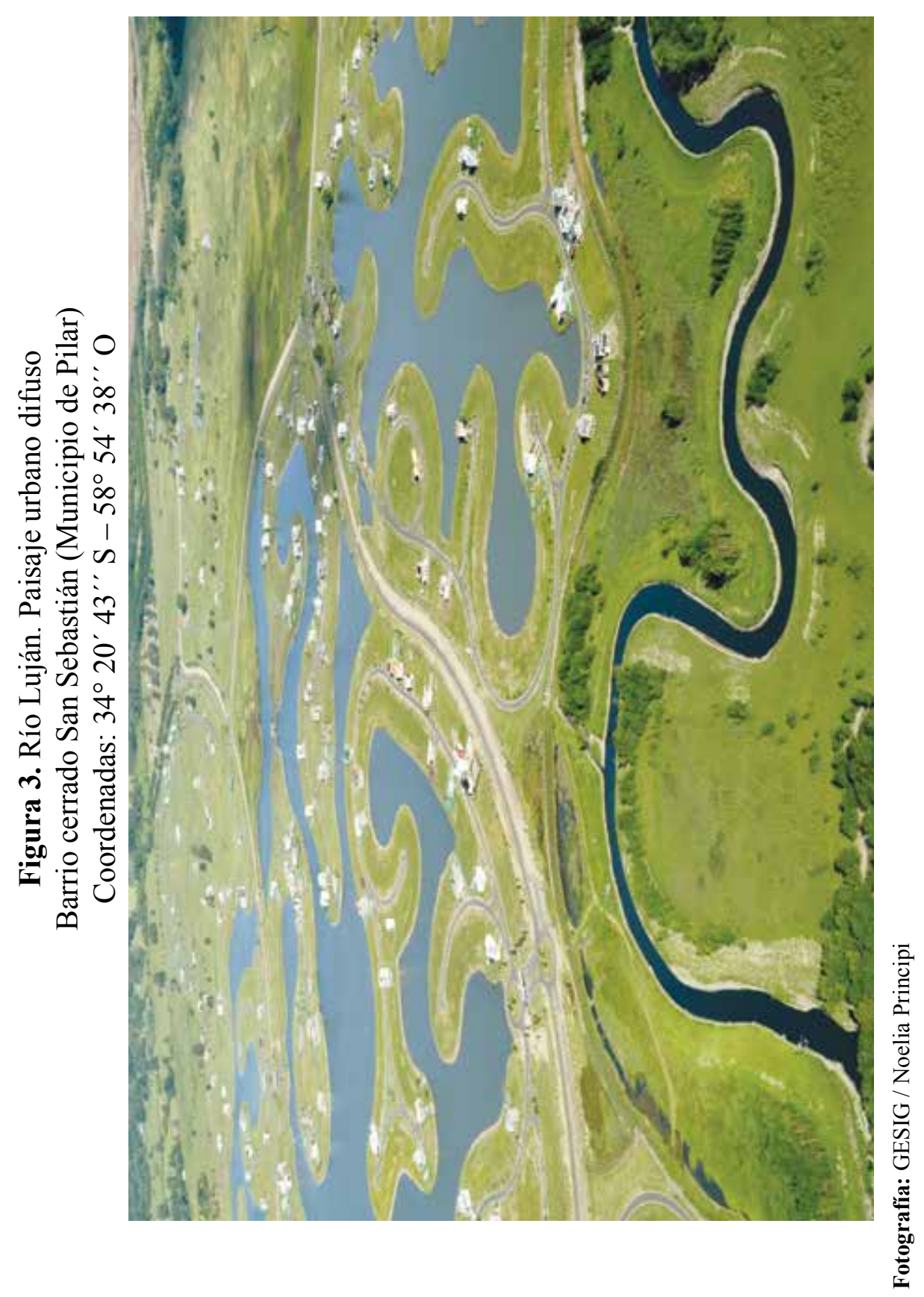


Gustavo D. Buzai, Noelia Principi. Identification of potential areas of land-use conflict in the Lujan river basin, Argentina

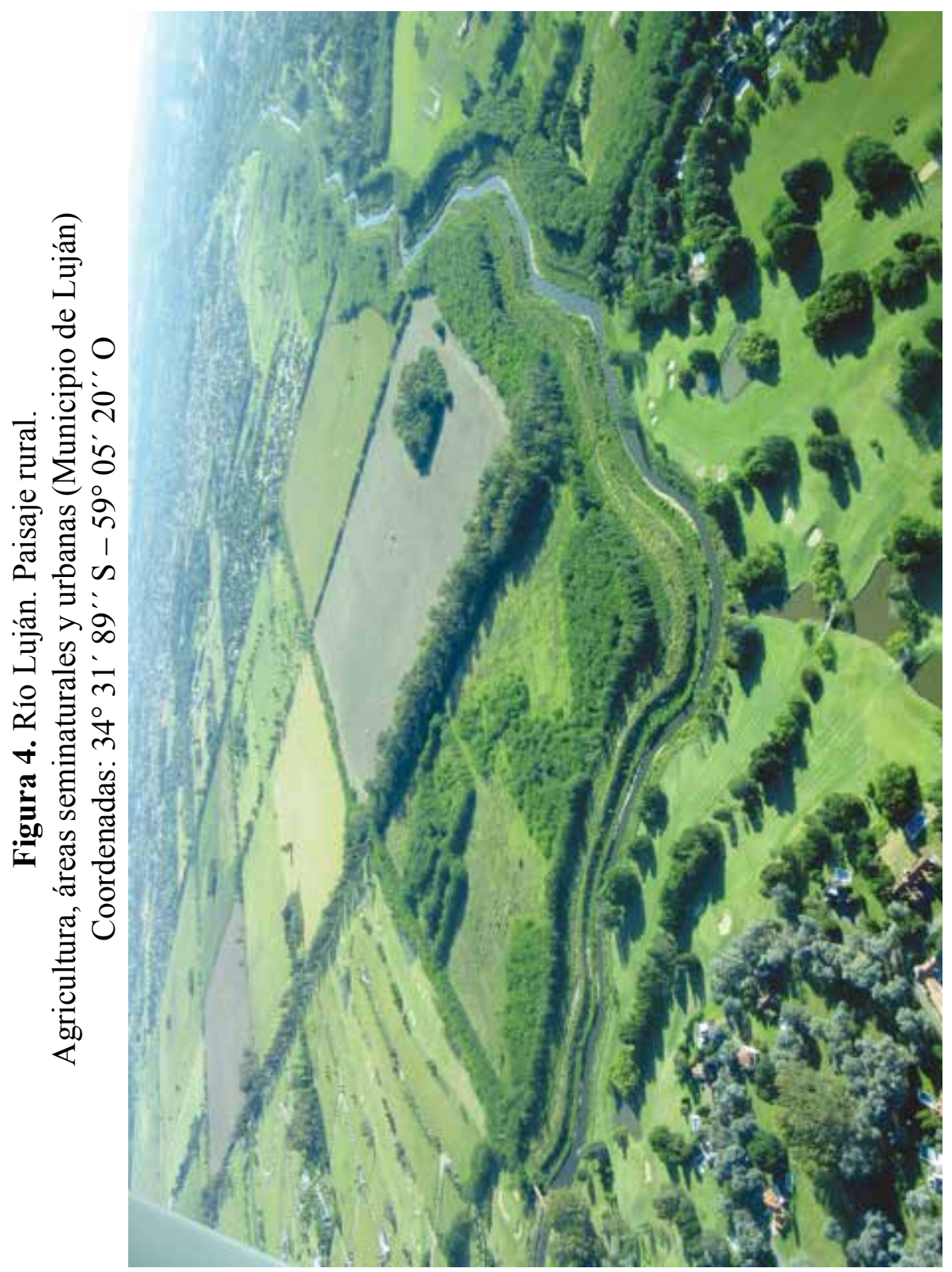




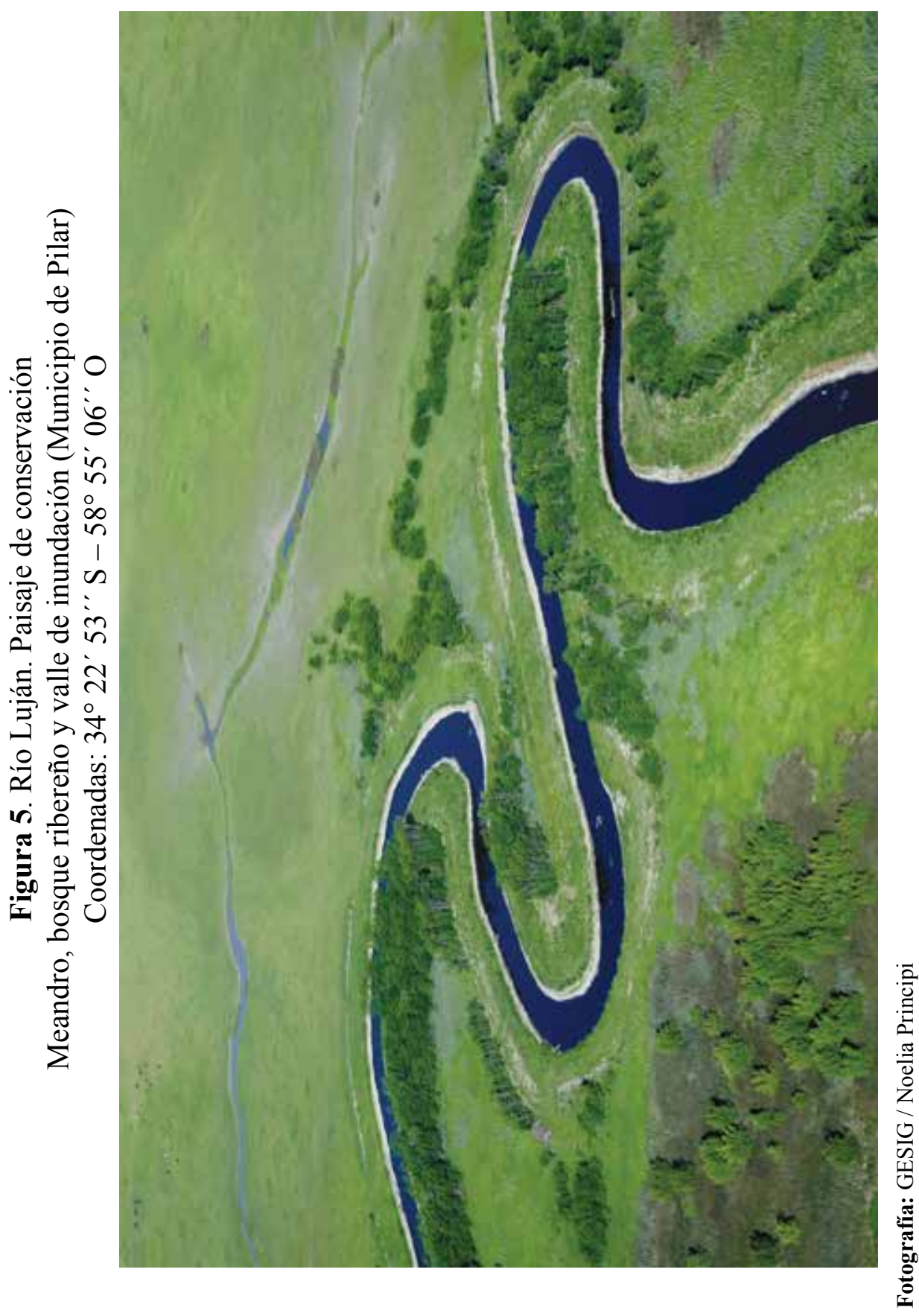


Gustavo D. Buzai, Noelia Principi. Identification of potential areas of land-use conflict in the Lujan river basin, Argentina

\section{Marco teórico-conceptual}

\section{Sistemas y materialismo sistémico}

Las distribuciones espaciales no se producen de manera aleatoria, sino que son resultado de acciones generales que llevan a la formulación de leyes científicas que explican la organización territorial. Desde un punto de vista sistémico, la Geografía se entiende como la ciencia que estudia las leyes que rigen las pautas de distribución espacial. Estas serán la base de los resultados obtenidos en el marco de una Geografía Aplicada (Pacione, 1999; Phlipponneau, 2001) en apoyo a la intervención en el ordenamiento territorial.

El considerar estas leyes permite realizar predicciones teóricas acerca de las distribuciones espaciales, en un claro ejemplo de lo que sería una Geografía Aplicada prospectiva que tiende al descubrimiento de relaciones de causalidad, pudiendo modelar configuraciones espaciales presentes y futuras.

Las investigaciones científicas en general apuntan a estudiar no sólo objetos específicos, sino que tienen particular interés sus relaciones. De esta manera, la perspectiva científica aborda totalidades al considerar la realidad como sistema.

Un marco de abordaje lo brinda la Teoría General de los Sistemas (TGS), formulada por Bertalanffy (1968) como construcción intelectual global que avanza empíricamente en el estudio de elementos y relaciones, con el objetivo central de encontrar las similitudes estructurales entre diferentes sistemas. De esta manera, se ponen en evidencia aspectos matemáticos que pueden ser utilizados para estudiar y describir comportamientos en diferentes escalas (Buzai y Cacace, 2016), desde una célula hasta una galaxia, encontrándose la escala humana en un nivel intermedio.

En un sistema no es posible explicar elementos aislados, sino que adquieren su total magnitud cuando se relacionan diferentes elementos en el marco de la estructura global que los vincula. La TGS permite unir las piezas separadas que produce la especialización científica y brinda posibilidades globales de análisis, existiendo un avance posterior en la búsqueda de especificidades.

El abordaje de aspectos específicos llevará a centrar la atención en diferentes escalas en la búsqueda de complementaciones. Esta posibilidad llegó a través de la Teoría de los Sistemas Complejos (TSC), formulada 
por García (2006), con base en los trabajos realizados en Epistemología Genética por Jean Piaget.

La TSC demostró importantes capacidades en dos niveles principales; en el estudio de la organización territorial y en la construcción de conocimientos con fundamento en su aptitud epistemológica (Piaget y García, 1983; García, 1997).

Estas capacidades resultan posibles, porque concibe a la realidad como una organización estratificada a partir de escalas con organizaciones semi-autónomas. Al aplicar conceptos de la TSC al estudio de la organización territorial, se realizan abordajes que podrían desembocar en tres niveles principales, uno focal que sería el de resolución propia de la temática en un nivel espacial, uno supra-focal de mayor generalidad y uno infrafocal de mayor nivel de detalle. De esta manera, existe una importante estabilidad conceptual, ya que pueden existir teorías específicas para cada nivel de la realidad.

La TGS se focaliza en aspectos generales y la TSC incluye aspectos específicos. Ambas brindan la posibilidad de estudiar la realidad como totalidad en sus múltiples dimensiones y detalles. El nivel de análisis espacial es el de la Geografía Aplicada, la focalización principal está puesta en la búsqueda de soluciones espaciales a problemáticas sociales, y para ello nuestra perspectiva es la del materialismo sistémico presentado por Bunge (2012, 2014).

Desde esta perspectiva, la Geografía Aplicada se basa actualmente en el análisis espacial cuantitativo con SIG y su focalización es empírica en un nivel regional (Baxendale. 2007) con una gran variedad de posibilidades aplicativas (Principi, 2013; Principi y Buzai, 2016). Para lograrlo su hilo conductor parte de considerar (Bunge, 2012) que la realidad existe (realismo), que puede ser estudiada a partir de sus elementos materiales (materialismo), que estos elementos se relacionan ampliamente en la conformación de sistemas (sistemismo) y que la forma más eficiente de acceder a ello es a través de la ciencia (cientificismo).

Llevando estas consideraciones a nuestra tarea de investigación, podemos apoyar estos cuatro aspectos a partir de considerar que la cuenca del río Luján, delimitada a través de la línea divisoria de aguas, existe independientemente del observador, que su contenido puede ser estudiado a partir de los elementos materiales que llevan a la definición de usos, de que 
estos usos tienen vínculos sistémicos que permiten llegar a resultados mediante el estudio de sus distribuciones y asociaciones espaciales y, finalmente, que la ciencia, a través de los SIG y del análisis espacial, permite modelizar este recorte de la realidad como totalidad organizada y obtener resultados que son de utilidad para actuar sobre la realidad empírica.

\section{Marco metodológico}

\section{Análisis de evaluación multicriterio}

La lógica del modelado de secuencias en SIG, para la búsqueda de localizaciones óptimas en diversas escalas, tiene su base inicial en la descomposición del área de estudio en un nivel vertical, formado por diferentes capas temáticas (layers) que contienen las variables fundamentales (temas en forma de mapas) útiles para la resolución.

Existen variados procedimientos que pueden ser aplicados a esta estructura de datos espaciales, principalmente incluidas en lo que se ha denominado modelado cartográfico, especialidad con mayor aptitud para las estructuras raster (Tomlin, 1990; DeMers, 2002) y que define las reglas de aplicación para la obtención de resultados mediante procedimientos de superposición cartográfica. El mayor avance en el modelado cartográfico lo constituyen las denominadas técnicas de evaluación multicriterio, presentadas con amplitud en Gómez Delgado y Barredo Cano, (2006) y Buzai y Baxendale (2011).

El análisis de evaluación multicriterio comienza con una información básica en formato cartográfico que sirve como criterio para realizar los procedimientos de evaluación. Hay dos tipos de criterios; aquellos que presentan valores continuos de aptitud locacional en cada variable, llamados factores, y las capas temáticas que actúan con la finalidad de confinar los resultados en un sector delimitado del área de estudio, llamados restricciones.

Cabe destacar aquí, que en la formación de factores pueden ser utilizadas las lógicas de tratamiento difuso, estandarizaciones fuzzy para la definición de zonas de aptitud sin límites precisos (Burrough y McDonnell, 1998; Jiang y Eastman, 2000) y, que según Wilson y Burrough (1999) podría ser considerada una de las nuevas bases para el análisis espacial cuantitativo.

Mediante la selección de variables, sus tratamientos tendientes hacia la generación de factores y restricciones, y la determinación de diferentes formas de combinación, nos encaminamos hacia la búsqueda de 
resultados. Las formas de vinculación se denominan reglas de decisión y su proceso de aplicación evaluación.

En Buzai y Baxendale (2011), se detallan estas relaciones junto a la utilización del triángulo de decisiones estratégicas (Figura 6), formado por un espacio de relaciones en el interior de dos ejes ortogonales (eje $x$ : nivel de riesgo, eje $y$ : nivel de compensación) y las soluciones posibles entre los métodos booleanos por multiplicación (análisis de riesgo mínimo sin compensación), suma (escalonamiento hacia el riesgo máximo sin compensación) y la combinación lineal ponderada (análisis de riesgo medio con máxima compensación).

Figura 6. Triángulo de decisiones estratégicas (locacionales)

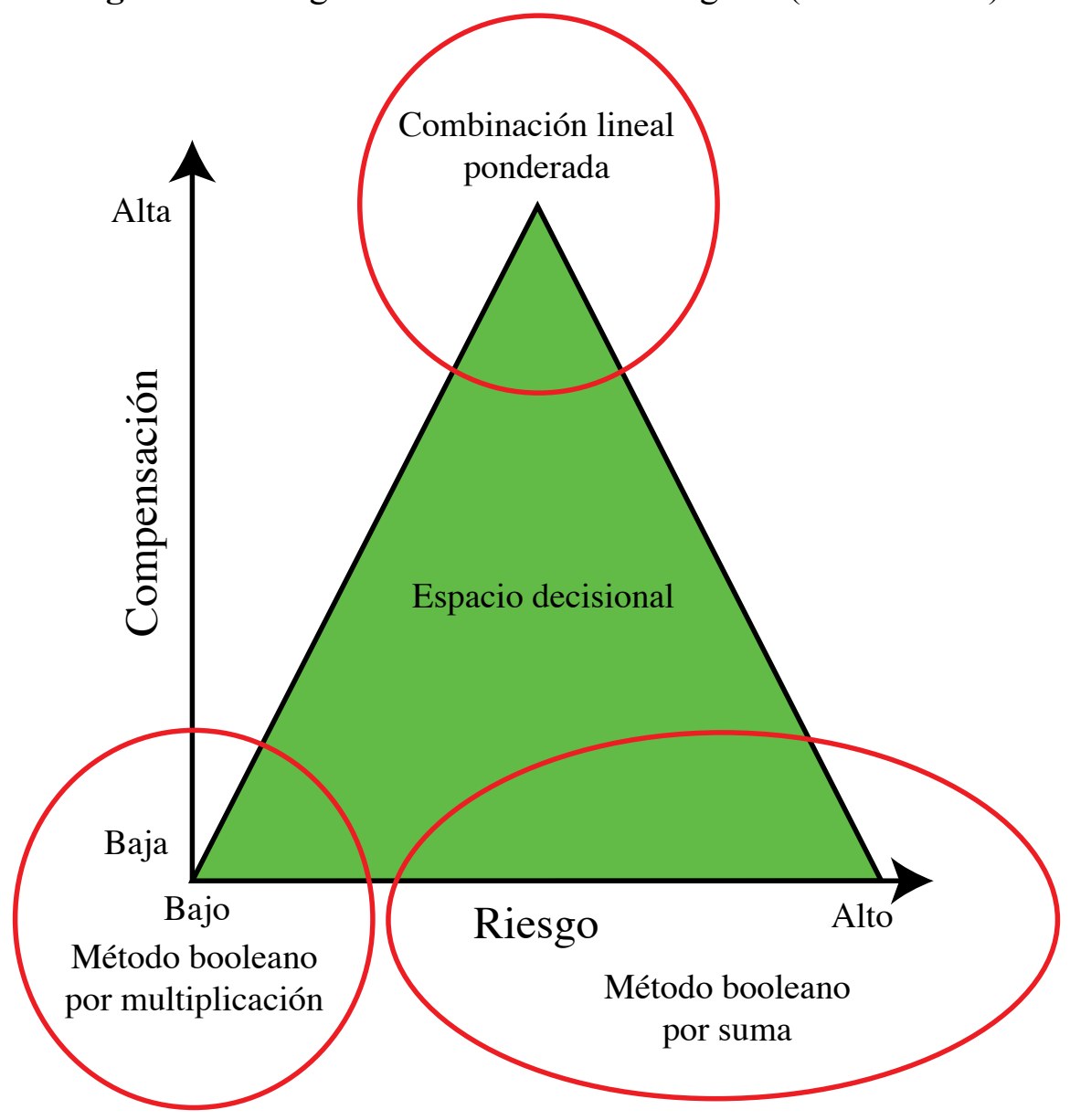


Gustavo D. Buzai, Noelia Principi. Identification of potential areas of land-use conflict in the Lujan river basin, Argentina

\section{Modelo de resolución}

Tomando como base la lógica de las técnicas de evaluación multicriterio, apoyadas por el uso de SIG, Carr y Zwick $(2006,2007)$ han propuesto un modelo de resolución estandarizada de interesantes capacidades para la identificación empírica de áreas potenciales de conflicto entre usos del suelo.

El modelo propuesto, que ha sido denominado LUCIS, contempla la realización de diferentes pasos como camino de resolución hacia la obtención del mapa con las áreas de conflicto. Algunos trabajos que analizan el método son los de Buzai y Baxendale $(2010,2011)$ y UF (2014), estudios previos en el área de estudio se encuentran en Buzai. Baxendale, Cacace y Dzendoletas (2011), y la presente aplicación fue realizada por Principi (2016) en el marco de un proyecto de investigación científica de reconocimiento nacional. ${ }^{5}$ Un avance actual fue realizado desde un punto de vista de la automatización a partir de la actualización realizada por Zwick, Patten y Arafat (2016), en la cual se presenta un nuevo aplicativo en SIG.

Los pasos para la aplicación del modelo son los siguientes:

1. Definición de objetivos: Se considera que cada localización puede tener tres posibilidades de ocupación en cuanto a usos, de los cuales pueden estar dedicados a actividades urbanas, agrícolas o de conservación. Cada una de estas actividades tiene como meta la maximización de oportunidades en ciertas líneas de desarrollo, por ejemplo, el uso urbano en cuanto a la expansión residencial y actividades de la producción secundaria y terciaria, el uso agrícola para la expansión de la producción primaria, principalmente la agricultura, o el uso de conservación para la protección ecológica.

2. Creación de la base de datos espacial: Se realiza, generando una serie de capas temáticas (layers) por digitalización que contienen información relevante y básica para el logro de cada uno de los tres objetivos señalados. Además de los temas a ser considerados en el análisis, en este punto se deben definir los aspectos técnicos de la creación de bases de

5 Proyecto: Análisis espacial y evaluación de zonas de potenciales conflictos ambientales, productivos y patrimoniales ante la expansión urbana en la cuenca del río Luján (Provincia de Buenos Aires, Argentina). Director: Dr. Gustavo Buzai, Codirectora: Dra. Sonia L. Lanzelotti. Proyecto de investigación científica y tecnológica (PICT-1388-2014) de la Agencia Nacional de Promoción Científica y Tecnológica (ANPCyT) de la República Argentina. 
datos alfanuméricas y gráficas en el marco de la tecnología SIG, como la extensión del área de estudio, sistema de proyección y unidad mínima de resolución espacial.

3. Análisis de aptitud: Se realiza el análisis de cada capa temática del área de estudio, determinando la aptitud relativa de las categorías de cada criterio para cada objetivo. Corresponde a la creación de factores para la aplicación de las técnicas de evaluación multicriterio. El método LUCIS considera una estandarización de aptitud continua (ac) en números enteros cumpliendo el siguiente rango:

$$
[1] 1 \leq a c \leq 9
$$

4. Determinación de preferencias: Se establece la importancia de cada factor en la resolución del objetivo específico. Para lograr el resultado se apela al conocimiento teórico o se utiliza una metodología para la obtención de los números representativos de la importancia de cada factor, en este estudio para nuestro caso fue aplicado el método de ponderación por ranking recíproco (Malczewski, 1999). El procedimiento de asignación de importancias puede estar apoyado por la colaboración entre expertos en cada temática involucrada (Jankowski y Nyerges, 2001) y, matemáticamente cumple las siguientes características:

$$
\begin{aligned}
& {[2] \sum p_{i}=1} \\
& {[3] 0<p_{i} \leq 1}
\end{aligned}
$$

5. Aplicación de la regla de decisión y reclasificación de resultados: Se ejecuta el procedimiento de combinación lineal ponderada, obteniéndose un valor índice sintético para cada localización en base a la definición de factores y los valores de ponderación para cada uno de ellos de la siguiente forma:

$$
\text { [4] } I_{i}=\sum p_{i} x_{i}
$$


o integrando restricciones espaciales incorporando la multiplicatoria de capas booleanas

$$
\text { [ } 5] I_{i}=\sum p_{i} x_{i} \Pi r_{j}
$$

El resultado corresponde a tres mapas de aptitud general para usos de urbano, agrícola o de conservación. Una reclasificación lleva cada valor de aptitud continua (ac) a tres categorías de la siguiente forma:

$$
\text { [6] } 1 \leq a c \leq 3
$$

El resultado corresponde a tres mapas de aptitud reclasificada para cada objetivo en aptitudes alta (A), media (M) y baja (B).

6. Identificación de áreas potenciales de conflicto: La combinación de usos conflictivos y no-conflictivos se encuentra en la combinatoria de las tres categorías para cada objetivo a partir de un gráfico de dispersión tridimensional que presenta las 27 combinaciones posibles de los tres mapas de aptitud, considerados el Objetivo 1, Objetivo 2 y Objetivo 3 (Figura 7). Los espacios que generan conflicto en la asignación de usos son los siguientes: $\mathrm{BBB}, \mathrm{MBM}, \mathrm{ABA}, \mathrm{MMB}, \mathrm{BMM}, \mathrm{MMM}, \mathrm{AMA}, \mathrm{AAB}, \mathrm{AAM}$, BAA, MAA y AAA destacados en color más oscuro. 
Figura 7. Combinación de objetivos y determinación de situaciones de potencial conflicto entre usos del suelo

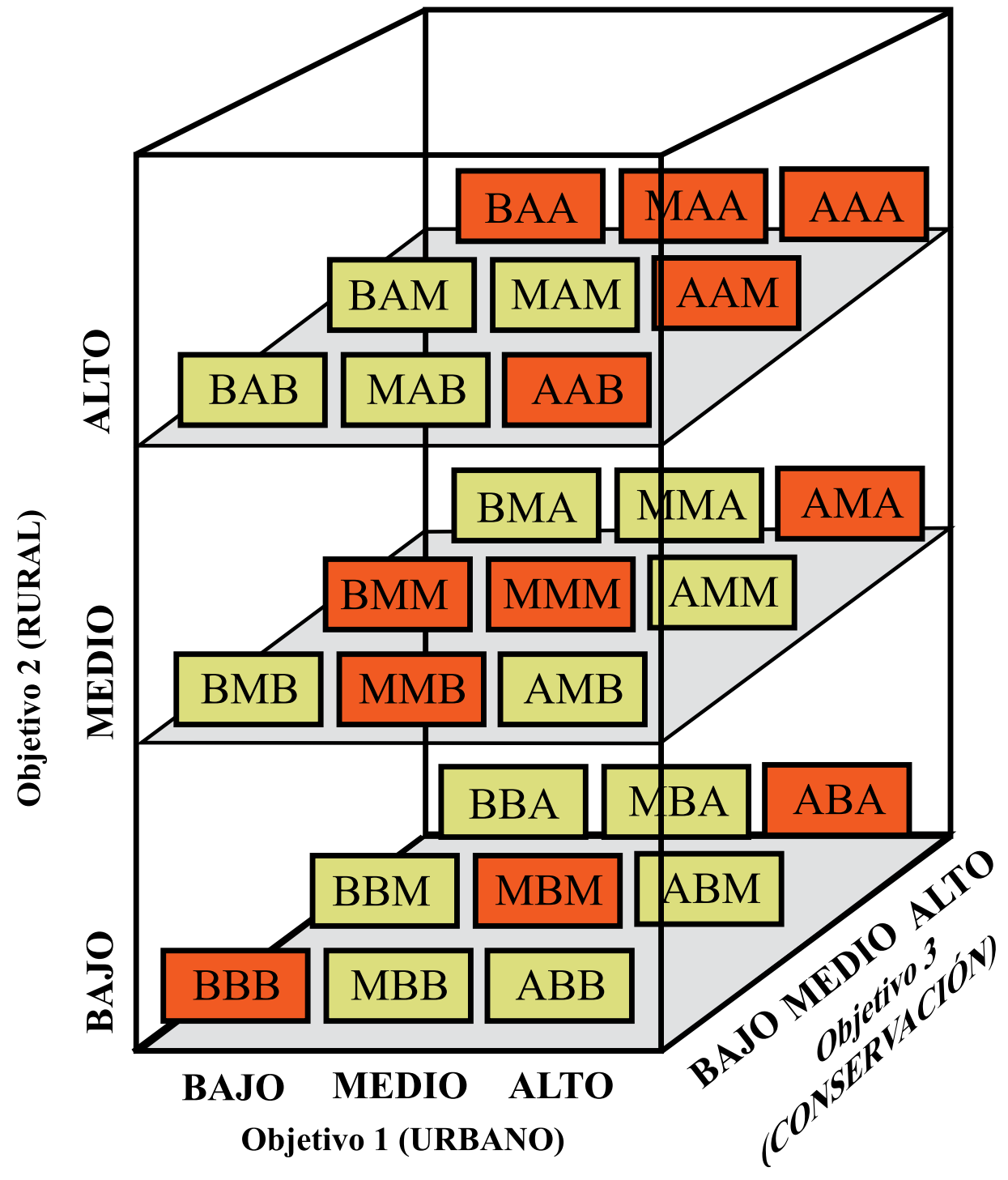

Fuente: Elaboración propia. 
Gustavo D. Buzai, Noelia Principi. Identification of potential areas of land-use conflict in the Lujan river basin, Argentina

\section{Resultados \\ Hacia la obtención de los mapas de aptitud Aptitud urbana}

La aptitud de cada localización para la expansión urbana ha sido calculada a partir de la utilización de 5 capas temáticas, en las cuales fueron aplicados los siguientes procedimientos para la creación de factores:

(a) Áreas urbanas. Consideración de áreas urbanas actuales y cálculo de distancias, aplicación de cálculo fuzzy lineal decreciente (255-0 en distancias de 0 a 2000 metros).

Criterio: Distancia a las áreas urbanas

(b) Red vial. Consideración de red vial (incluye rutas nacionales y provinciales, caminos pavimentados y de tierra) y cálculo de distancias, aplicación de cálculo fuzzy lineal decreciente (255-0 en distancias de 0 a 3000 metros).

Criterio: Distancia a la red vial

(c) Estaciones de ferrocarril. Consideración de las estaciones de ferrocarril y cálculo de distancias, aplicación de cálculo fuzzy lineal decreciente (255-0 en distancias de 0 a 2000 metros).

Criterio: Distancia a estaciones de ferrocarril

(d) Bañados: terrenos bajos y húmedos, a trechos cenagosos y a veces inundados por aguas pluviales o por las de un río o laguna cercana. Consideración de los bañados como 0 , aplicación de cálculo fuzzy lineal decreciente (255-0 en distancias 0 a 10000 metros de los bañados).

Criterio: Presencia de bañados

(e) Cursos de agua. Consideración de los cursos de agua, cálculo de un buffer de distancia de 1000 metros y luego cálculo de distancias, aplicación de cálculo fuzzy lineal decreciente (255-0 en distancias de 0 a 2000 metros considerando el buffer).

Criterio: Distancia a cursos de agua 
El método de ponderación consideró el siguiente ordenamiento en la importancia de los factores: áreas urbanas (1), red vial (2), cursos de agua (3), bañados (3) y estaciones de ferrocarril (4), brindando como resultado los valores 0,$41 ; 0,21 ; 0,14 ; 0,14$ y 0,10 respectivamente. Como restricciones fue utilizado el mapa de áreas urbanas actuales.

El mapa de aptitud urbana presenta una reclasificación del resultado en tres categorías, base para la combinación posterior, correspondiente al objetivo 1 que ocupa el eje $x$ en la Figura 7. 
Gustavo D. Buzai, Noelia Principi. Identification of potential areas of land-use conflict in the Lujan river basin, Argentina

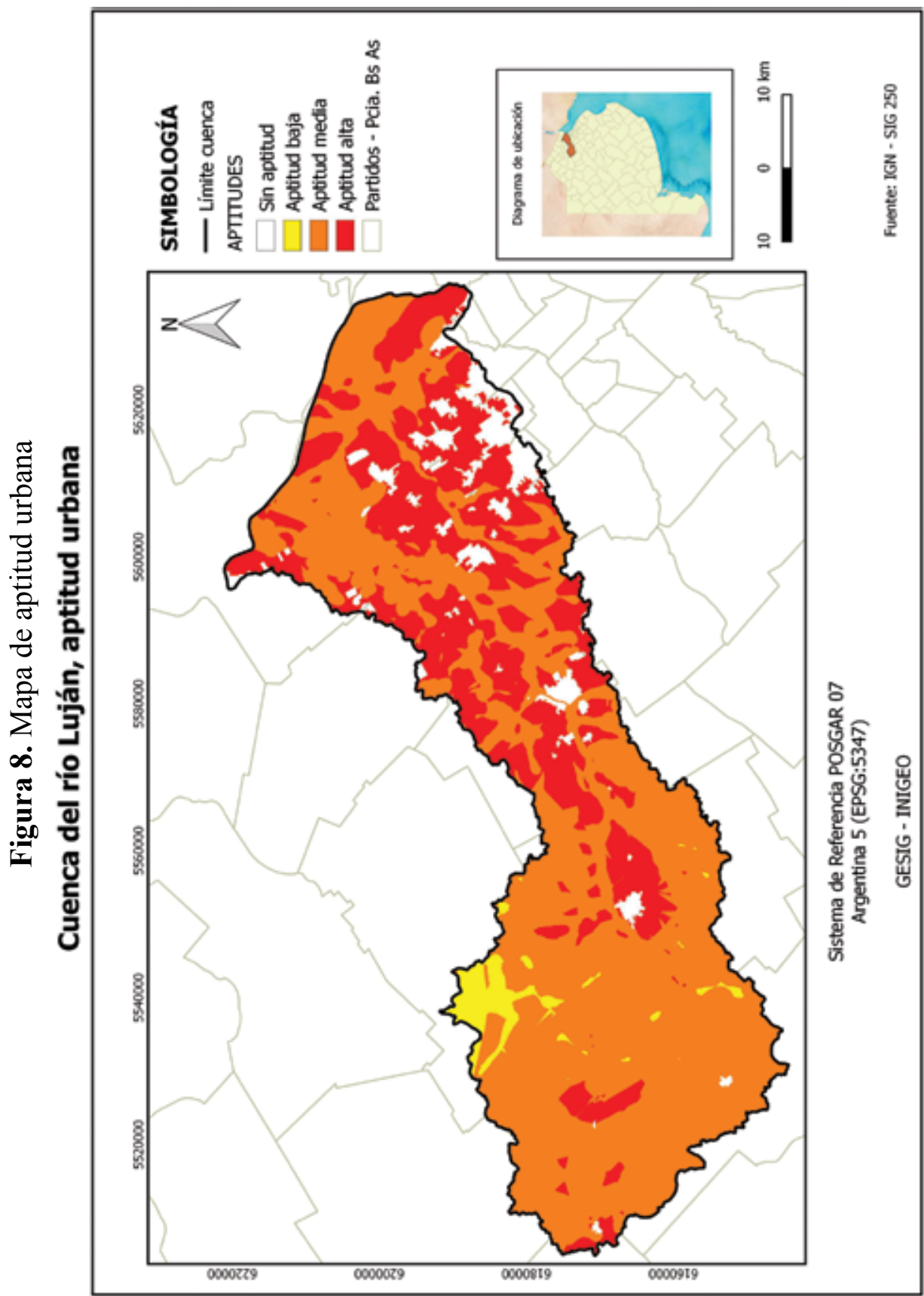

110 Revista Geográfica de América Central No 59

ISSN 1011-484X, julio-diciembre 2017, pp. 91-124 


\section{Aptitud Agrícola}

La aptitud de cada localización para la expansión de la actividad agrícola ha sido calculada a partir de la utilización de 3 capas temáticas, en las cuales fueron aplicados los siguientes procedimientos para la creación de factores:

(a) Relieve (Categorías: 0-10m, 10-20m, 20 -30m, 30-40m, 40-50m y 50-60m). Aplicación de cálculo fuzzy lineal decreciente (255-0 la cobertura de mayor aptitud en la categoría de mayor altura)

Criterio: Altura del terreno

(b) Suelos (Categorías: uso de tierra mixto; plantaciones perennes; bosque artificial; cañadas; bañados; ciénaga, tremedal, tembladeral; paleocauce o cauce abandonado; cordones o acumulación de conchillas y planta urbana). Reclasificación en el siguiente orden: 1, 2, 3, 4, 5, 6, 7, 8 y 9 respectivamente. Aplicación de cálculo fuzzy lineal decreciente (255-0 la cobertura de mayor aptitud en la primera categoría)

Criterio: Coberturas del suelo

(c) Infraestructura rural. Consideración de la infraestructura rural existente y cálculo de distancias, aplicación de cálculo fuzzy lineal decreciente (255-0 en distancias de 0 a 4000 metros).

Criterio: Cercanía a la infraestructura rural existente

La aplicación del método de ponderación consideró el siguiente ordenamiento en la importancia de las capas temáticas: Relieve (1), Suelos (1), Infraestructura rural (3), brindando como resultado los valores 0,43 ; 0,43 y 0,14 respectivamente.

El mapa de aptitud urbana presenta una reclasificación del resultado en tres categorías, base para la combinación posterior, correspondiente al objetivo 2 que ocupa el eje $y$ en la Figura 7. 
Gustavo D. Buzai, Noelia Principi. Identification of potential areas of land-use conflict in the Lujan river basin, Argentina

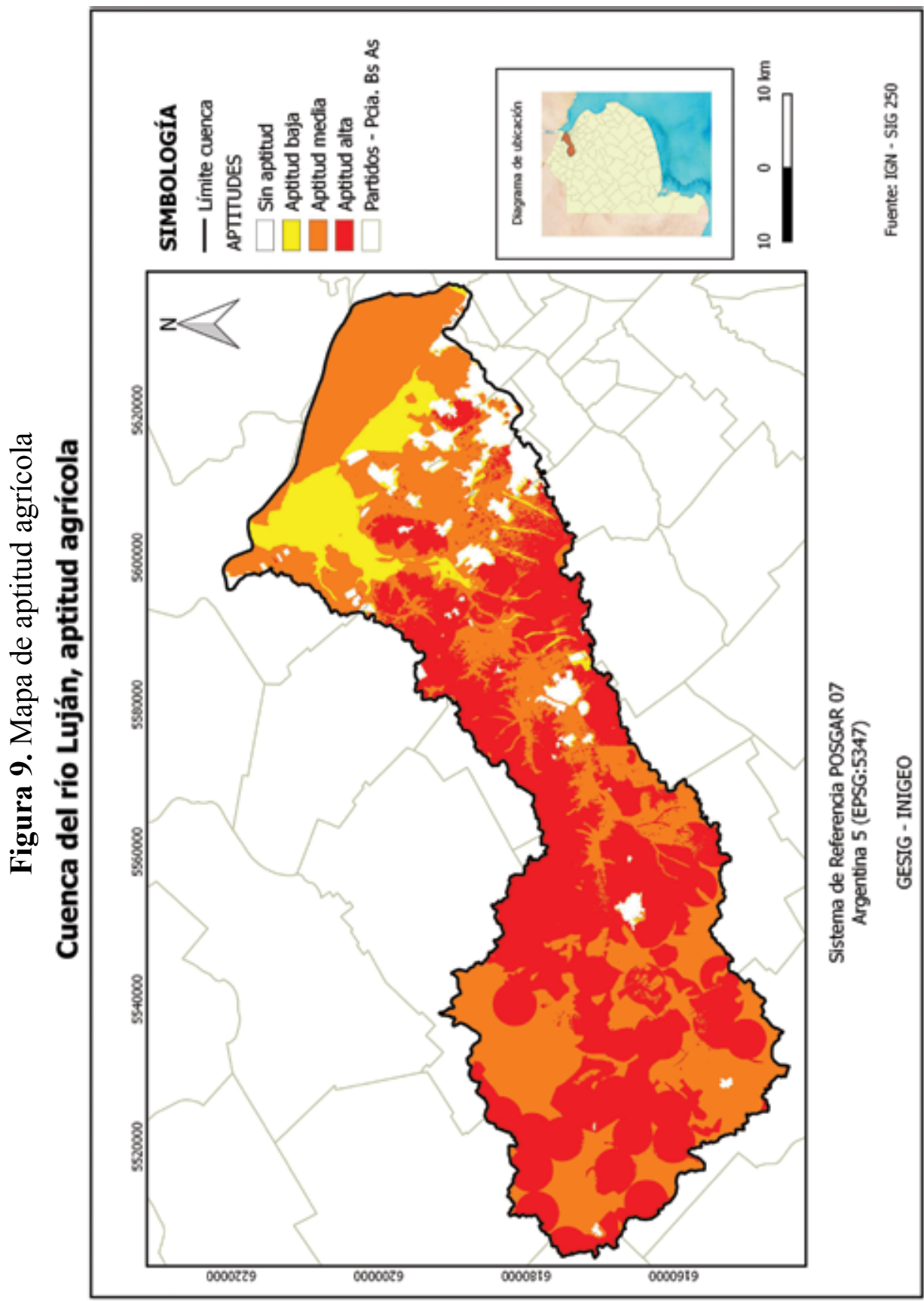




\section{Aptitud para la conservación}

La aptitud de cada localización para su conservación ha sido calculada a partir de la utilización de 3 capas temáticas, de las cuales fueron derivados 3 factores a partir de la aplicación de los siguientes procedimientos para la creación de factores:

(a) Suelos. (Categorías: bañados; cañadas; paleocauce o cauce abandonado; ciénaga, tremedal, tembladeral; cordones o acumulación de conchillas; bosque artificial; plantaciones perennes, uso de tierra mixto y planta urbana). Reclasificación en el siguiente orden: 1, 2, 3, 4, 5, 6, 7, 8 y 9 respectivamente. Aplicación de cálculo fuzzy lineal decreciente (255-0 la cobertura más óptima la primera categoría).

Criterio: Coberturas del suelo

(b) Cursos de agua. Consideración de los cursos de agua y cálculo de distancias, aplicación de cálculo fuzzy lineal decreciente (255-0 en distancias de 0 a 1000 metros).

Criterio: Distancia a cursos de agua

(c) Cuerpos de agua. Consideración de los cuerpos de agua y cálculo de distancias, aplicación de cálculo fuzzy lineal decreciente (255-0 en distancias de 0 a 1000 metros).

Criterio: Distancia a cuerpos de agua

La aplicación del método de ponderación consideró el siguiente ordenamiento en la importancia de las capas temáticas: Suelos (1), Cursos de agua (2) Cuerpos (2), brindando como resultado los valores 0,$50 ; 0,25$ y 0,25 respectivamente.

El mapa de aptitud urbana presenta una reclasificación del resultado en tres categorías, base para la combinación posterior, correspondiente al objetivo 3 que ocupa el eje $z$ en la Figura 7. 
Gustavo D. Buzai, Noelia Principi. Identification of potential areas of land-use conflict in the Lujan river basin, Argentina

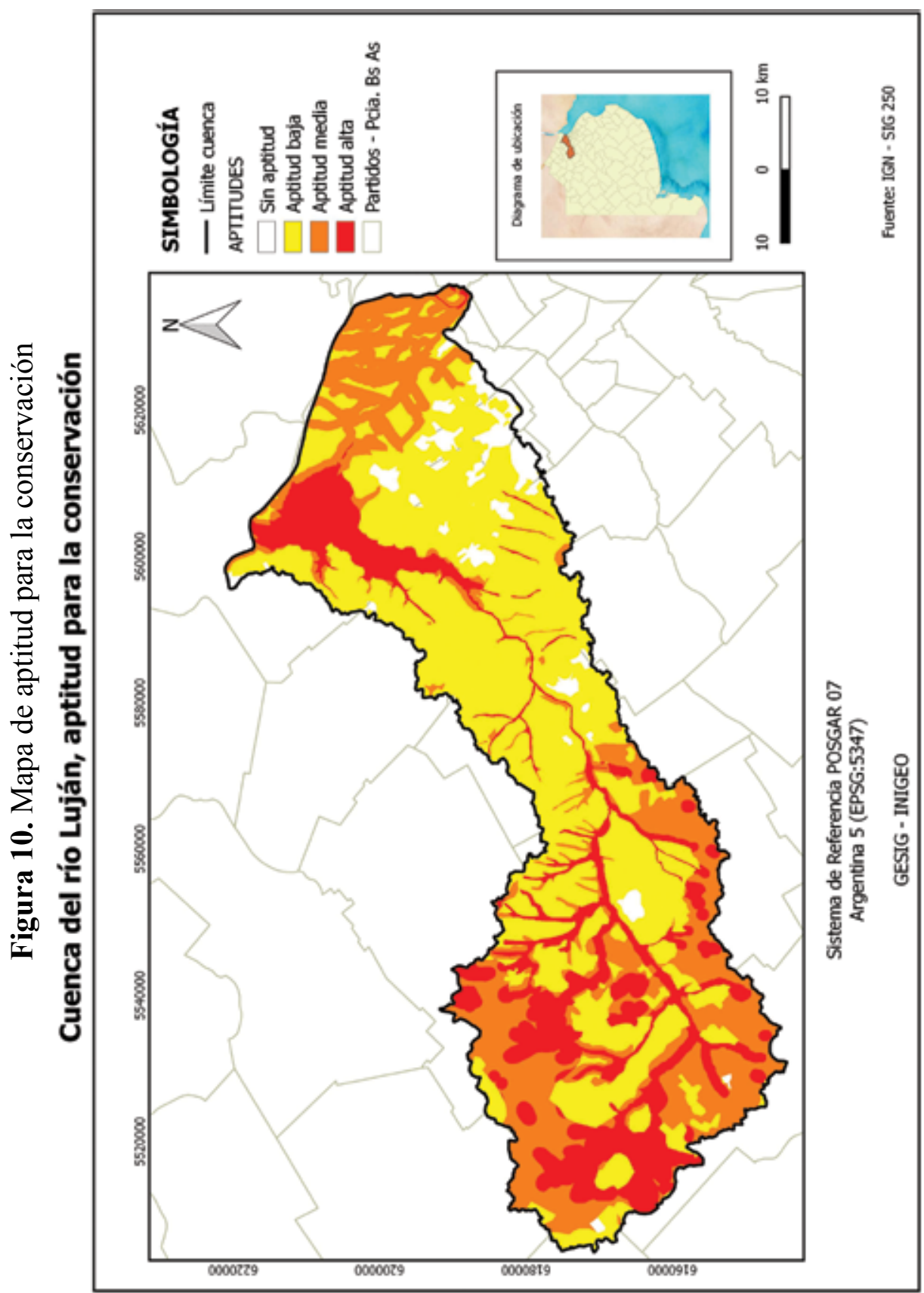

114 Revista Geográfica de América Central No 59

ISSN 1011-484X, julio-diciembre 2017, pp. 91-124 


\section{Hacia la obtención del mapa de potenciales conflictos entre usos del suelo}

La síntesis de la tarea metodológica realizada se encuentra plasmada en el diagrama de solución (Figura 11), en el cual se detalla la secuencia que lleva al resultado final del Mapa de potenciales conflictos entre usos del suelo.

Figura 11. Diagrama de Solución

EVALUACIÓN MULTICRITERIO

LUCIS

Criterios para el logro de objetivos

Objetivos

(resultados parciales)

Combinación de objetivos

(resultado final)

Distancia a áreas urbanas $(0,41)$

Distancia a red vial $(0,21)$

Distancia a estaciones de ferrocarril $(0,14)$

Presencia de bañados $(0,14)$

Distancia a cursos de agua $(0,10)$

Altura del terreno $(0,43)$

Cobertura del suelo $(0,43)$

Distancia a infraestructura rural $(0,14)$

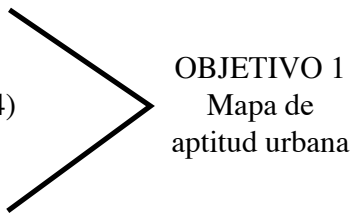

Cobertura del suelo $(0,50)$

Distancia a cursos de agua $(0,25)$

Distancia a cuerpos de agua $(0,25)$

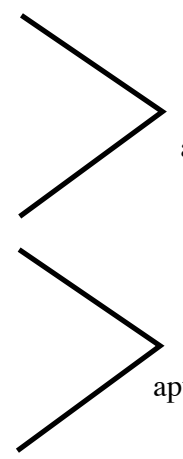

OBJETIVO 2

Mapa de aptitud agrícola

OBJETIVO 3

Mapa de aptitud conservación

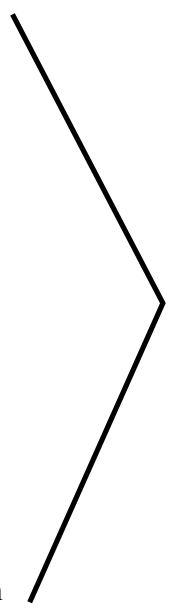

Mapa de potenciales conflictos entre usos del suelo

La identificación de áreas se realiza a través de la combinatoria de categorías de los mapas presentados anteriormente como objetivos parciales (urbano, agrícola y conservación).

El mapa aptitud urbana fue reclasificado en centenas (los valores pasaron de 1 a 100, de 2 a 200 y de 3 a 300), el mapa Aptitud Agrícola fue reclasificado en decenas (los valores pasaron de 1 a 10, de 2 a 20 y de 3 a 30) y el mapa Aptitud Conservación quedó con sus valores originales (1, 2 y 3 ).

Al sumar las tres capas temáticas de aptitud (con 3 categorías cada una: 1-baja, 2-media y 3-alta) se produce una nueva capa temática que puede llegar a tener un total de 27 combinaciones. A continuación, se 
presentan estas combinaciones divididas en aquellas que producen áreas de potencial conflicto (12 resultados) y en las que no (15 resultados).

Las combinaciones de categorías con potencial conflicto son: (111) Conflicto urbano-agrícola-conservación*, (122) Conflicto agrícola conservación, (133) Conflicto agrícola -conservación, (212) Conflicto urbano-conservación, (221) Conflicto urbano- agrícola, (222) Conflicto urbano- agrícola -conservación*, (233) Conflicto agrícola-conservación, (313) Conflicto urbano-conservación, (323) Conflicto urbano-conservación, (331) Conflicto urbano- agrícola, (332) Conflicto urbano- agrícola, y (333) Conflicto urbano- agrícola -conservación * 6

Las combinaciones de categorías sin potencial conflicto son: (112) Localización conservación, (113) Localización conservación, (121) Localización agrícola, (123) Localización conservación, (131) Localización agrícola, (132) Localización agrícola, (211) Localización urbana, (213) Localización conservación, (223) Localización conservación, (231) Localización agrícola, (232) Localización agrícola, (311) Localización urbana, (312) Localización urbana, (321) Localización urbana, y (322) Localización urbana

Los resultados obtenidos para la cuenca del río de Luján se presentan en el Cuadro 1 y su agregación en categorías genéricas en el Cuadro 2:

$6 \quad$ El asterisco (*) indica un conflicto mayor, aquellas localizaciones donde existe similar aptitud en las tres categorías. Las otras combinaciones conflictivas se producen entre dos categorías. 
Cuadro 1. Resultados combinatorios desagregados de áreas con potencial conflicto entre usos del suelo en la Cuenca del Rio Luján

\begin{tabular}{|l|l|l|l|}
\hline Combinación & \multicolumn{1}{|c|}{ Aptitudes dominantes } & Superficie $\left.\mathbf{( K m}^{\mathbf{2}}\right)$ & Superficie (\%) \\
\hline 111 & Urbano-Agrícola-Conservación & No se produce & - \\
\hline 122 & Agrícola-Conservación & 35,02 & 0,93 \\
\hline 133 & Agrícola-Conservación & 13,67 & 0,36 \\
\hline 212 & Urbano-Conservación & 27,92 & 0,74 \\
\hline 221 & Urbano-Agrícola & 188,43 & 5,01 \\
\hline 222 & Urbano-Agrícola-Conservación & 562,05 & 14,95 \\
\hline 233 & Urbano-Conservación & 203,57 & 5,41 \\
\hline 313 & Urbano-Conservación & 13,33 & 0,35 \\
\hline 323 & Urbano-Conservación & 8,37 & 0,22 \\
\hline 331 & Urbano-Agrícola & 516,19 & 13,72 \\
\hline 332 & Urbano-Agrícola & 7,41 & 0,20 \\
\hline 333 & Urbano-Agrícola-Conservación & 1,98 & 0,05 \\
\hline Total & Conflictos completos & 1577,94 & 41,95 \\
\hline
\end{tabular}

Cuadro 2. Resultados combinatorios agrupados de áreas con potencial conflicto entre usos del suelo en la Cuenca del Rio Luján

\begin{tabular}{|l|l|l|l|}
\hline Combinación & \multicolumn{1}{|c|}{ Aptitudes dominantes } & Superficie $\left.\mathbf{( K m}^{\mathbf{2}}\right)$ & Superficie (\%) \\
\hline $122-133-233$ & Agrícola-Conservación & 252,26 & 6,71 \\
\hline $212-313-323$ & Urbano-Conservación & 49,62 & 1,31 \\
\hline $221-331-332$ & Urbano-Agrícola & 712,03 & 18,93 \\
\hline $222-333$ & Urbano-Agrícola- Conservación & 564,03 & 14,99 \\
\hline Total & Conflictos completos & 1577,94 & 41,95 \\
\hline
\end{tabular}

El mapa síntesis, obtenido a través del método LUCIS, presenta la distribución espacial de los potenciales conflictos presentados en el Cuadro 2. 
Gustavo D. Buzai, Noelia Principi. Identification of potential areas of land-use conflict in the Lujan river basin, Argentina

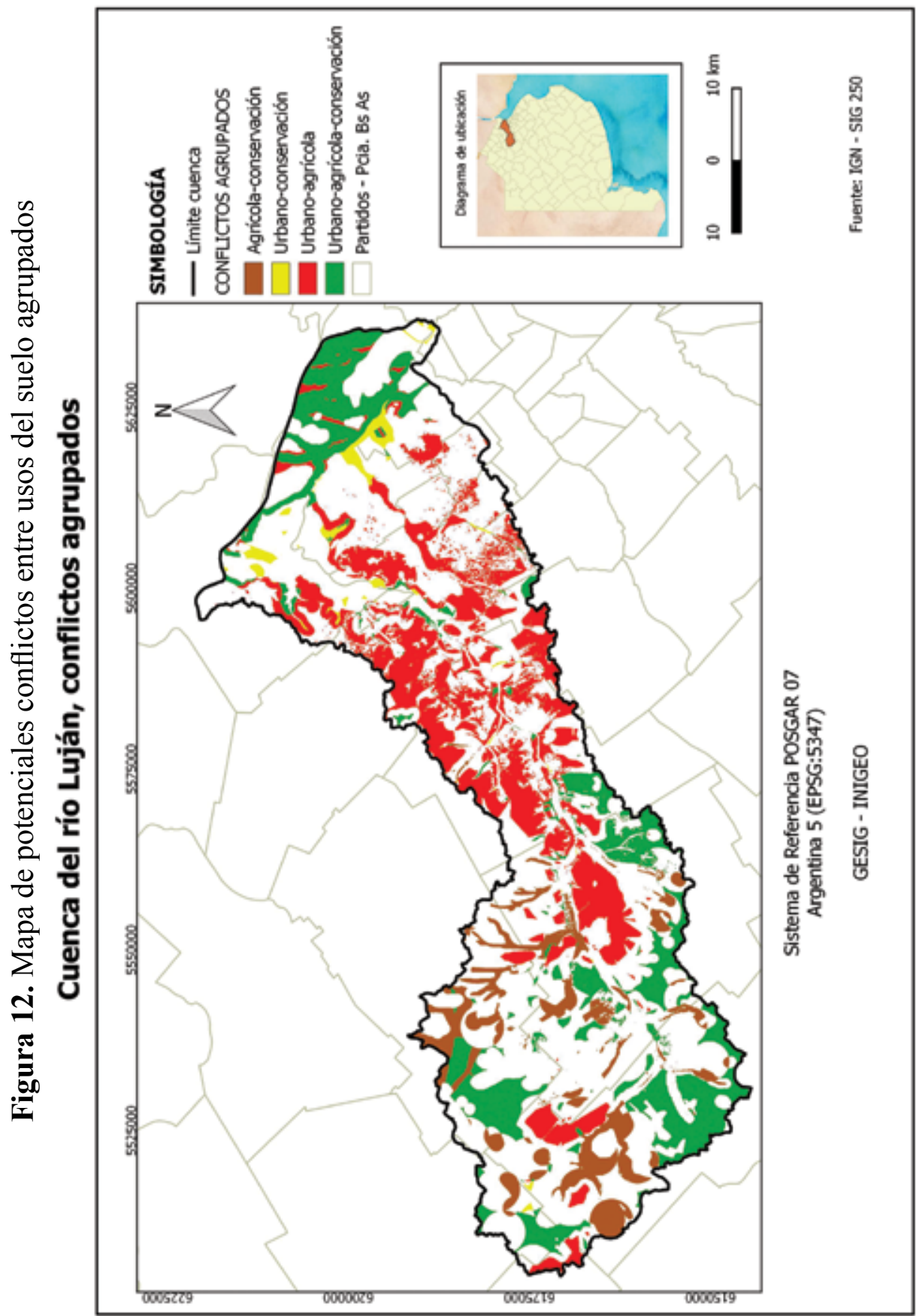




\section{Conclusiones}

La síntesis de la tarea metodológica realizada a partir del modelado de secuencias, adoptado en la investigación, fue presentada secuencialmente en los diferentes puntos de este trabajo. Las etapas corresponden a la definición de criterios, obtención de mapas de aptitud para cada objetivo parcial y el resultado final, con asociaciones espaciales que presentan la base de interpretación.

Los cuadros presentados brindan los valores de superficie de potenciales conflictos como aproximación cuantitativa a su magnitud.

Los valores agregados muestran la síntesis obtenida, y la Figura 12 presenta las distribuciones espaciales como base para su obtención. La combinación urbano-agrícola resulta ser la mayor área de potencial conflicto con una superficie de $712,03 \mathrm{~km}^{2}$ distribuidos por la totalidad de la cuenca y con predominio en su sector central. Le sigue la combinación urbano-agrícola-conservación con $564,03 \mathrm{~km}^{2}$ distribuidos, principalmente, en los extremos correspondientes a la cuenca inferior (zona de delta) y a la superior. La conflictividad potencial entre agrícola-conservación, con $252,26 \mathrm{~km}^{2}$, se encuentra principalmente en la cuenca superior y entre urbano-conservación con 49,62 $\mathrm{km}^{2}$ en la inferior.

El valor superficial total de potencial conflicto es de $1577,94 \mathrm{~km}^{2}$, lo que corresponde a un $41,95 \%$ de la cuenca del río Luján que tiene una superficie total de $3761 \mathrm{~km}^{2}$. De ese porcentaje, el 45,12\% lo concentra el conflicto urbano-agrícola. Los resultados obtenidos responden a una situación esperable para una región con una gran dinámica regional. Se encuentran zonas plenamente urbanas y rurales entre un gran sistema periurbano, correspondiente a la expansión del Gran Buenos Aires en estructura de urbanización dispersa y el crecimiento compacto de ciudades de tamaño intermedio. Se localiza la presencia de muchos de los suelos más fértiles del país como sustento de una importante producción agrícola, y también la de importantes cuerpos de agua en un mosaico de paisajes apto para la preservación de la diversidad.

Finalmente, podemos afirmar que las técnicas de evaluación multicriterio se han convertido en una de las más interesantes líneas de aplicación de la tecnología SIG como herramienta de planificación territorial, particularmente en su posibilidad de realizar diagnósticos y propuestas de intervención. 
Gustavo D. Buzai, Noelia Principi. Identification of potential areas of land-use conflict in the Lujan river basin, Argentina

Basado en ellas, el método LUCIS presenta una serie de procedimientos que ligan aspectos cuantitativos y cualitativos, procurando la identificación de áreas de potencial conflicto en cuanto a la expansión de usos del suelo. El producto obtenido para la cuenca del río Luján ha mostrado la aptitud teórico-metodológica de los procedimientos aplicativos. Los resultados presentan una configuración espacial de mucha utilidad para la toma de decisiones en la planificación regional, su regulación ambiental o la orientación del crecimiento urbano. Las posibilidades de transferencia de estos hacia los niveles de gestión político-administrativa ${ }^{7}$ constituyen una etapa posterior con tendencia a presentar utilidad de lo obtenido como aporte de la Geografía Aplicada orientada a la aplicación de conocimientos geográficos orientados a la resolución de problemáticas socioespaciales.

\section{Referencias}

Baxendale, C.A. (2007). Región y estudios regionales. Consideraciones desde los diferentes enfoques de la Geografía [Region and Regional Studies. Considerations from Different Perspectives of Geography]. Fronteras. 6(6), 29-36.

Bertalanffy, L.von. (1968). General System theory: Foundations, Development, Applications. New York: George Braziller.

Bunge, M. (2012). A la caza de la realidad [Hunting the Reality]. Barcelona: Gedisa.

Bunge, M. (2014). Memorias. Entre dos mundos [Memories. Between Two Worlds]. Buenos Aires: EUDEBA.

Burrough, P.A., McDonnell, R.A. (1998). Principles of Geographical Information Systems. Oxford: Oxford University Press.

Buzai, G.D. (Dir.) (2002). Altas digital de la cuenca del río Luján [Digital Atlas of Luján River Basin]. Luján: Universidad Nacional de Luján. (CD version).

Buzai, G.D., Baxendale, C.A. (2007). Áreas de potencial conflicto entre usos del suelo. Identificación mediante el uso de Sistemas de

7 La Universidad Nacional de Luján mantiene convenios de cooperación y transferencia con el Comité de Cuenca del Río Luján (COMILU) y con los municipios integrantes. Asimismo, es una de las unidades académicas integrantes de la red interinstitucional universitaria UNIDESARROLLO, integrada por las universidades de las cuencas del río Luján y Reconquista. Hacia estos ámbitos institucionales son dirigidos los resultados de los proyectos de investigación que toman el espacio regional como área de estudio. 
Información Geográfica (Primera parte: descripción metodológica) [Areas of Potential Conflict between Land Uses. Identification through the Use of Geographic Information Systems (First Part: Methodological Description)]. Fronteras. 6(6), 45-49.

Buzai, G.D., Baxendale, C.A. (2008). Áreas de potencial conflicto entre usos del suelo. Identificación mediante el uso de Sistemas de Información Geográfica (Segunda parte: aplicación) [Areas of Potential Conflict between Land Uses. Identification through the Use of Geographic Information Systems (Second Part: Application)]. Fronteras. $7(7), 33-39$.

Buzai, G.D., Baxendale, C.A. (2010). Método LUCIS [LUCIS Method] Land Use Conflict Identification Strategy. Geografia y Sistemas de Información Geográfica [Geography and Geographic Information Systems]. (Bibliografía, Software y Metodología). 2(2),1-4.

Buzai, G.D., Baxendale, C.A. (2011). Análisis Socioespacial con Sistemas de Información Geográfica [Socio-Spatial Analysis with Geographic Information Systems]. Volume 1: Perspectiva cientifica temáticas de base vectorial [Scientific Perspective-Vectorial Base Topics]. Buenos Aires: Lugar Editorial.

Buzai, G.D., Baxendale, C.A., Cacace, G., Dzendoletas, M.A. (2011). Análisis de usos del suelo urbano y regional. Localizaciones óptimas y conflictivas estudiadas con Sistemas de Información Geográfica [Analysis of uses of Urban and Regional Soils. Best and Conflictive Localizations studied with Geographic Information Systems]. PROEG 11. Luján: Universidad Nacional de Luján.

Buzai, G.D., Cacace, G. (2016). El concepto de espacio [The concept of Space]. In: Buzai, G.D., Baxendale, C.A.; Humacata, L., Cacace, G., Delfino, H., Lanzelotti, S., Principi, N. Geografia y Análisis Espacial. Aplicaciones urbano-regionales con Sistemas de Información Geográfica [Geography and Spatial Analysis. Urban-Regional Applications with Geographic Information Systems]. (51-56). Luján: EdUNLu.

Carr, M.H.; Zwick, P.D. (2006). Using GIS suitability analysis to identify potential future land use conflicts in north central Florida. Journal of Conservation Planning. 1(1), 89-105.

Carr, M.H.; Zwick, P.D. (2007). Smart Land-Use Analysis. The LUCIS Model. ESRI Press. Redlands. 
DeMers, M. (2002). GIS Modeling in Raster. New York: John Wiley. García, R. (Coord) (1997) La Epistemología Genética y la ciencia contemporánea [Genetic Epistemology and the contemporary Science]. Barcelona: Gedisa.

García, R. 2006. Sistemas Complejos [Complex Systems]. Barcelona: Gedisa. Gómez Delgado, M., Barredo Cano, I. (2006). Sistemas de Información Geográfica y evaluación multicriterio en la ordenación del territorio [Geographic Information Systems and Multicriteria Evaluation in the Spatial Planning]. México DF: Alfaomega-Ra-Ma..

Instituto Nacional del Agua [National Institute of Water]. (2007). Diagnóstico del funcionamiento hidrológico hidráulico de la cuenca del río Luján - provincia de Buenos Aires [Diagnosis of the Hydrological Functioning of Luján River Basin-Buenos Aires Province]. Ministerio del Interior, Obras Públicas y Vivienda [Ministry of the Interior, Public Works and Housing]. Ezeiza.

Jankowski, P.; Nyerges, T. (2001). GIS-Supported Collaborative Decision Making: Results of an Experiment. Annals of the Association of American Geographers. 91(1),48-70.

Jiang, H., Eastman, J.R. (2000). Application of fuzzy measures in multicriteria evaluation in GIS. International Journal of Geographical Information Sciences. 14(2), 173-184.

Lanzelotti, S.L.; Buzai, G.D. (2015). Delimitación de la cuenca del río Luján, Provincia de Buenos Aires Argentina [Delimitation of Luján River basin, Buenos Aires Province, Argentina]. Informe Técnico $\mathrm{N}^{\circ}$ 1 [Technical Report N 1]. PICT-2014_1388. GESIG-PRODISIG. Luján: Universidad Nacional de Luján.

Malczewski, J. (1999). GIS and multicriteria decision analysis. New York: John Wiley \& Sons.

Pacione, M. (1999). Applied Geography: Principles and Practice. London: Routledge.

Paso-Viola, L.F. (2015) Análisis cronológico documental de la evolución de la cartografía en la alta cuenca del río Luján bajo la óptica de los procesos histórico globales y locales. Diagnóstico cartográfco geonímico, toponímico y geoespacial del Partido de Suipacha como modelo casuístico [Chronological Documentary Analysis of Cartography Evolution in the Luján River High Basin Under the 
Perspective of Global and Local Historic Processes. Cartographic, Geonimic, Toponimic and Geo-Spatial Diagnose of Suipacha Partido as Casuistic Model]. (Tesis de Licenciatura en Geografía. Universidad Nacional de Luján [Bachelor's degree thesis in Geography. National University of Luján]).

Paso-Viola, L.F. (2016). Origen del Río Luján en el área imbrífera localizada en los partidos de Chacabuco, Suipacha y Carmen de Areco y su representación cartográfica [Luján River Origins in the Drainage Basin of Chacabuco, Suipacha and Carmen de Areco and their Cartographic Representation]. Social Networks. 3(1),17-30.

Philipponneau, M. (2001). Geografía Aplicada [Applied Geography]. Barcelona: Ariel.

Principi, N. (2013). Análisis de la estructura espacial regional [Analysis of the Regional Spatial Structure]. Editorial Académica Española: Saarbrücken.

Principi, N. (2016). Evaluación multicriterio para la identificación de áreas con potenciales conflictos entre usos del suelo en la cuenca del río Luján [Multicriteria Evaluation for the Identification of Areas with Potential Conflicts between Land Uses in the Luján River Basin] (Buenos Aires, Argentina). (Trabajo final de graduación. Maestría Profesional en Sistemas de Información Geográfica y Teledetección [Master's Paper. Master Degree in Geographic Information Systems and Remote Sensing]. UCR-UNA. San José, Costa Rica)

Principi, N., Buzai, G.D. (2016). Análisis especial para la determinación de la estructura socioespacial del Noroeste de la Provincia de Buenos Aires [Special Analysis for the Determination of the Socio-Spatial Structure of Nothwestern Region of Buenos Aires Province]. In: Buzai, G.D., Baxendale, C.A., Humacata, L., Cacace, G., Delfino, H., Lanzelotti, S., Principi, N. Geografía y Análisis Espacial. Aplicaciones urbano-regionales con Sistemas de Información Geográfica. [Geography and Spatial Analysis. Urban-Regional Applications with Geographic Information Systems] (197-204). Luján: EdUNLu. Tomlin, C.D. (1990). Geographic Information Systems and Cartographic Modeling. New Jersey: Englewood Cliffs - Prentice Hall..

UF .(2014). Land Use Conflict Identification Strategy (LUCIS). Technical Report. LUCIS Technical Report. Heartland 2060. University of Florida.. 
Wilson, J.P. \& Burrough, P.A. (1999). Dynamic Modeling, Geostatistics, and Fuzzy Classification: New Sneakers for a New Geography? Annals of the Association of American Geographers. 89,736-746.

Zwick, P.D.; Patten, I.E.; Arafat, A. (2016). Advanced Land-Use Analysis for Georegional Design. Using LUCISplus. Redlands: ESRI Press.

Wilson, J.P. \& Burrough, P.A. (1999). Dynamic Modeling, Geostatistics, and Fuzzy Classification: New Sneakers for a New Geography? Annals of the Association of American Geographers. 89,736-746.

Zwick, P.D.; Patten, I.E.; Arafat, A. (2016). Advanced Land-Use Analysis for Georegional Design. Using LUCISplus. Redlands: ESRI Press. 